\title{
Avaliação da incidência da SOP por clínica e ultrassonografia, em residentes na cidade de Paracatu - MG
}

\author{
Evaluation of the incidence of SOP by clinical and ultrasonography \\ in residents of the city of Paracatu - MG
}

\author{
Lethícia Silva Santos ${ }^{1}$, Anna Luisa Oliveira Garcia ${ }^{1}$, Guilherme Alves Souza ${ }^{1}$, \\ Jéssica Gonçalves Benevides ${ }^{1}$, Talitha Araújo Faria ${ }^{2}$
}

\begin{abstract}
Silva LS, Garcia ALO, Souza GA, Benevides JG, Faria TA. Avaliação da incidência da SOP por clínica e ultrassonografia, em residentes na cidade de Paracatu - MG / Evaluation of the incidence of SOP by clinical and ultrasonography in residents of the city of Paracatu $M G$. Rev Med (São Paulo). 2018 jul.-ago.;97(4):402-6.
\end{abstract}

RESUMO: A síndrome do ovário policístico é um distúrbio endócrino, causado pela presença de microcistos produtores de hormônio nos ovários, que afeta de 4 a 13\% das mulheres em idade reprodutiva. O hiperandrogenismo e a anovulação crônica estão presentes em praticamente todas as pacientes, sendo fatores essenciais para o fechamento do diagnóstico da SOP. A causa específica da síndrome é desconhecida, porém sabe-se que possui relação direta com a síndrome metabólica. O tratamento da SOP, envolve fármacos sensibilizadores de insulina, reguladores de hormônios femininos, e principalmente mudança de hábitos de vida. Assim, o presente trabalho objetivou avaliar a incidência de SOP por meio de clínica e ultrassonografia. Foi utilizado o método de coorte, onde os dados sobre a incidência da síndrome do ovário policístico foram levantados, em demanda examinada na cidade de Paracatu - MG, nos anos de 2009 ao primeiro semestre de 2016. Os casos de alterações em volumes uterinos e ovarianos foram maiores em adolescentes. A SOP, foi encontrada principalmente na faixa etária de 20 a 29 anos, o tipo de exame mais realizado nas pacientes foi o transvaginal. Identificou-se que as formações císticas mais comuns foram ovários com pequenos folículos esparsos na periferia e apenas 30 pacientes dentre as analisadas tiveram comorbidades associadas. Verificou-se que a SOP é mais presente na vida das adolescentes, porém o diagnóstico, na maioria, é tardio, o que favorece o não tratamento e pior qualidade de vidas às portadoras.

Descritores: Síndrome do ovário policístico; Ovário/crescimento e desenvolvimento; Utero/crescimento e desenvolvimento; Tamanho do órgão; Brasil/epidemiologia.

\begin{abstract}
Polycystic ovary syndrome is an endocrine disorder, caused by the presence of hormone-producing microcysts in the ovaries that affects 4 to $13 \%$ of women of reproductive age. Hyperandrogenism and chronic anovulation are present in practically all patients, being essential factors for closing the diagnosis of PCOS. The specific cause of the syndrome is unknown, but it is known to be directly related to the metabolic syndrome. The treatment of PCOS, involves insulin sensitizing drugs, regulators of female hormones, and mainly change of lifestyle. Thus, the present study aimed to evaluate the incidence of PCOS by means of clinical and ultrasonography. The cohort method was used, where the data on the incidence of polycystic ovary syndrome were collected, in a review examined in the city of Paracatu - MG, in the years 2009 to the first half of 2016 . The cases of changes in uterine and ovarian volume was higher in adolescents. PCOS was found mainly in the age group of 20 to 29 years, the type of examination most performed in the patients was the transvaginal. It was identified that the most common cystic formations were ovaries with small sparse follicles in the periphery and only 30 patients among those analyzed had associated comorbidities. It was found that PCOS is more present in adolescent life, but the diagnosis is mostly late, which favors non-treatment and poorer quality of life for the carriers.
\end{abstract}

Keywords: Polycystic ovary syndrome; Ovary/growth \& development; Uterus/growth \& development; Organ size; Brazil/ epidemiology.

\footnotetext{
*Trabalho apresentado na forma oral, no I Congresso Acadêmico de Medicina e Saúde da Faculdade Atenas, Paracatu, MG, em 20 de maio de 2016. 1. Acadêmicas(os) do Curso de Medicina do Centro Universitário Atenas (UniAtenas). ORCID: LS Santos - https://orcid.org/0000-0003-4835; ALO Garcia - https://orcid.org/0000-0001-9719-497X; GA Souza - https://orcid.org/0000-0002-5414-1349; JC Benevides - https://orcid.org/0000-0002-83173518. Emails: lethiciass@outlook.com, annaluisa-garcia@hotmail.com, guilherme.alvesouza@gmail.com, siicabenevides@hotmail.com. 2. MsC, Docente do curso de medicina da Faculdade Atenas. ORCID: https://orcid.org/0000-0002-4252-6794. Email: talithabio@yahoo.com.br. Endereço para correspondência: Lethícia Silva Santos. Rua José Mundim, 94, apt. 3. Centro, Paracatu, MG. Email: lethiciass@outlook.com.
} 


\section{INTRODUÇÃO}

Gíndrome é a associação de características, Sintomas, sinais e fenômenos que ocorrem simultaneamente, e a aparição de uma é fator condicionante para que outra surja ${ }^{1}$.

A síndrome do ovário policístico (SOP) é um distúrbio endócrino complexo que acomete de 4 a $13 \%$ das mulheres em idade reprodutiva. Está relacionada ao descontrole hormonal e metabólico heterogêneo, ou seja, tem relação com alterações hormonais e morfológicas em pessoas do sexo feminino. Costuma aparecer na adolescência em meninas na faixa etária de 10 a 19 anos, com o surgimento da puberdade, quando iniciam-se as mudanças hormonais que dão as características femininas, mas que, no caso da doença, também intensificam os sinais e sintomas típicos ${ }^{2-4}$.

A SOP foi descrita primeiramente por Stein e Leventhal, em 1935, quando observaram a associação entre a amenorreia, hirsutismo, obesidade e ovários em aspectos policísticos com volumes aumentados bilateralmente. Anos seguintes o termo síndrome do ovário policístico foi adotado. Com o aprimoramento tecnológico foi acrescido ao diagnóstico clínico e anatômico, o diagnóstico ultrassonográfico e bioquímico, assim como é atualmente ${ }^{1}$.

As características mais relacionadas com o distúrbio são a anovulação crônica, ausência de ovulação no período menstrual; aparição de microcistos nos ovários ou alteração de volume, o que nomeia a síndrome; e o hiperandrogenismo, excesso de hormônios andrógenos, causador e/ou intensificador de todos os sintomas relacionados a SOP. A partir de tais características é possível confirmar o diagnóstico segundo o critério de Rotterdam, em que dois dos sinais citados acima diagnosticam a síndrome ${ }^{3-6}$.

Os sintomas e sinais mais frequentes na síndrome são: a infertilidade; amenorreia, ausência de menstruação em três ciclos seguidos; o hirsutismo, crescimento anormal de pelos no corpo feminino, onde rotineiramente não há; aparecimento exacerbado de acnes; obesidade, principalmente a do tipo central; e síndrome metabólica ${ }^{5}$.

Os riscos associados estão ligados ao aparecimento da síndrome metabólica, que segundo a Sociedade Brasileira de Cardiologia ainda precisa de um conceito bem estabelecido, no entanto é reconhecida pelo aumento da pressão arterial, disfunção metabólica dos lipídeos e glicídios, excesso de peso e aumento de morbimortalidade cardiovascular (IAM, infartos agudos do miocárdio). Visto que associada a SOP pode gerar precocemente, antes da quarta década de vida, o DM2 (Diabetes Melitus tipo 2), devido à resistência insulínica; HAS (Hipertensão arterial sistêmica) também precoce, por causa do aumento da resistência periférica causada pela hiperinsulinemia; disfunção endotelial; marcadores anti-inflamatórios crônicos; obesidade central; dislipidemia; aumento na atividade fibrinolítica; hipercalcificação na artéria coronária $^{3,7,8}$.

É importante lembrar e reforçar que mesmo após uma gama de estudos ainda não foi possível determinar a causa da síndrome do ovário policístico, contudo sabe-se da conexão que ela possui com a síndrome metabólica. $\mathrm{O}$ tratamento da SOP atualmente está ligado, na maioria dos casos, ao uso de agentes sensibilizadores de insulina, dos quais o principal representante é a Metformina, que interfere no mecanismo da insulina, diminuindo-a. A insulina ativa receptores que aumentam a secreção androgênica, intensificando os sintomas encontrados na SOP, sendo então a Metformina uma droga importante na amenização dos distúrbios, além de a longo prazo prevenir a aparição da DM2. Outras drogas utilizadas são os anticoncepcionais, para regular parte dos hormônios femininos, diminuindo sintomas como acne, por exemplo. Porém vale ressaltar que o melhor tratamento para a SOP ainda é a mudança de hábitos como: abandono do sedentarismo, emagrecimento, alimentação regular e saudável ${ }^{6-11}$.

Segundo Costa et al. ${ }^{6}$, atualmente, existem poucos estudos que abordam a prevalência da síndrome metabólica em mulheres portadoras da SOP, sendo a maioria deles realizados na população feminina norte-americana, na qual a obesidade é observada como fator mais prevalente que nas europeias ou sul-americanas. Entretanto o conhecimento e a pesquisa da prevalência da síndrome metabólica podem ser determinantes na identificação de um subgrupo de pacientes portadoras da SOP, cujo risco de complicações cardiovasculares é mais elevado, o que justificaria uma abordagem direcionada à proteção endotelial.

Estudos constataram que a frequência da síndrome metabólica (SMet) foi aproximadamente duas vezes maior entre mulheres adultas com SOP do que em adolescentes com o distúrbio, embora o índice de massa corporal (IMC) tenha evidenciado relação com o aparecimento da SMet tanto na idade adulta quanto na adolescência de mulheres com SOP. Há relatos na literatura de frequências elevadas de SMet em adolescentes com SOP que não estão associadas à hiperinsulinemia, mesmo na presença de obesidade. No entanto, a SMet em adolescentes com SOP também foi demonstrada associada à obesidade ${ }^{9,12}$.

A SOP vem sendo estudada em várias faixas etárias com o intuito de diagnóstico e tratamento mais precoce possível. Dados de pesquisas realizadas no município de Viçosa - MG retratam que a prevalência de SOP em adolescentes entre 15 e 18 anos foi de $6,2 \%$. Das mulheres de 20 a 30 anos diagnosticadas com SOP, 76,5\% apresentaram HDL-C abaixo de $50 \mathrm{mg} / \mathrm{dl}$, evidência que pode relacionar com a síndrome dislipidêmica, logo com a SMet. Assim como o sedentarismo, hábito observado em $79,5 \%$ das diagnosticadas, que é visto como um agravante $^{2,3,6}$.

Com isso o presente trabalho objetivou avaliar a incidência da SOP, de forma a apresentar informações 
mais consistentes, e conjuntamente promover o diagnóstico precoce, tendo em vista maior qualidade de vidas às portadoras da síndrome.

\section{MÉTODOS}

O trabalho científico é do tipo observacional, descritivo com delineamento de coorte, com base em dados primários, recolhidos em estabelecimentos de atenção secundária à saúde especializados no atendimento à mulher do município de Paracatu, no período entre 2009 ao primeiro semestre de 2016. Os dados reuniram todas as mulheres, de várias idades, residentes em Paracatu, que possuíam pelo menos um dos critérios de inclusão que eram: ovários policísticos previamente diagnosticados, história clínica sugestiva, hiperandrogenismo e ou hiperandrogenenia. Não foram utilizados os prontuários das pacientes selecionadas, tendo sido a amostra universo agrupada a partir das confirmações supracitadas. Os valores de referências das faixas etárias estão baseados nos liberados pela OMS, sendo adolescentes meninas de 10 a 19 anos; adultas, mulheres de 20 a 49 anos; e por fim menopausadas com mais de 50 anos. Enquanto os valores de referências usados como padrão de comparação dos volumes ovarianos estão descritos na Coletânea de tabelas aplicadas em ultrassonografia ${ }^{14}$.

Usando-se de um tratamento estatístico por averiguação de dados foi calculado teste de associação risco relativo, e programas Word (2013) e Excel (2013) para confecção de gráficos e tabelas abordando quantitativamente os dados encontrados.

\section{RESULTADOS}

Para a realização do estudo foi utilizado dados de 255 mulheres do município de Paracatu - MG, correspondendo ao universo de diagnósticos no local de pesquisa e período amostral pré-determinado. Sendo possível encontrar em seus exames ultrassonográficos particularidades e generalidades sobre a síndrome, em relação às faixas etárias. Destas, 39 apresentaram alguma comorbidade ginecológica associada. Sendo $15,38 \%$ casos com mioma uterino, $23,07 \%$ com aumento considerável no ovário esquerdo, $43,58 \%$ com aumento surpreendente nos ovários direitos, $5,12 \%$ com varizes uterinas, 7,69\% com ovários atrofiados; $2,56 \%$ com várias áreas com cicatrizes; e por fim $2,56 \%$ com cistos consideravelmente grandes na região paraovariana, e ainda há uma mulher usuária de DIU.

Para os volumes encontrados calculou-se a média e estes foram comparados aos volumes normais máximos de cada estrutura anatômica.

A média do volume uterino com alteração, em relação às idades, foi mais prevalente nas adolescentes, com $62,6 \mathrm{~cm}^{3}$ (Gráfico 1).
Gráfico 1- Média dos volumes uterinos das pacientes analisadas

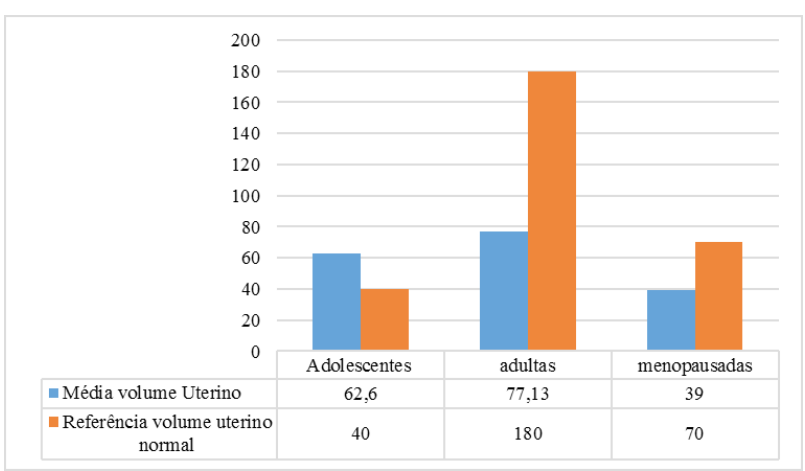

A média do volume ovariano direito com alteração, em relação às idades, foi mais prevalente nas adolescentes, com $16,44 \mathrm{~cm}^{3}$ (Gráfico 2).

Gráfico 2. Média dos volumes dos ovários direitos das pacientes analisadas

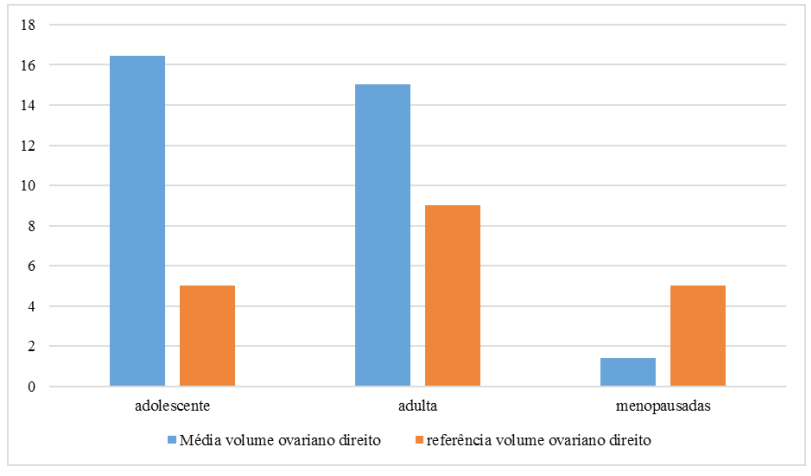

A média do volume ovariano esquerdo com alteração, em relação às idades, foi mais prevalente nas adolescentes, com 15,39 $\mathrm{cm}^{3}$ (Gráfico 3).

Gráfico 3. Média dos volumes dos ovários esquerdos das pacientes analisadas

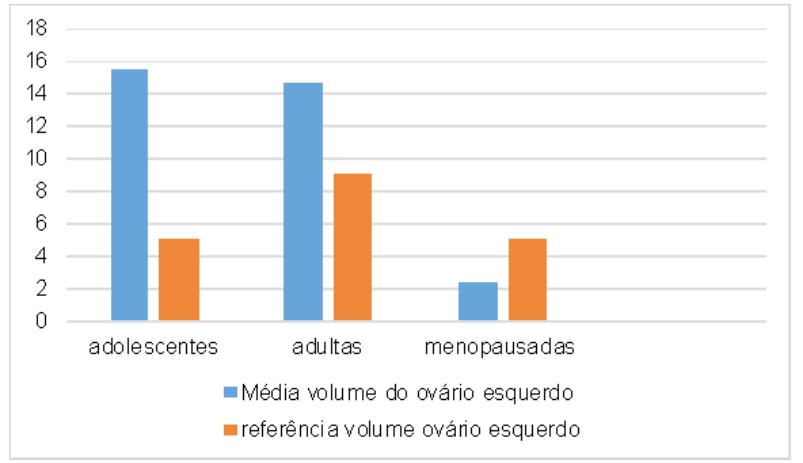


A porcentagem referente às idades com alteração foi mais prevalente na faixa etária entre 20 e 49 anos, em que 104 mulheres foram encontradas (Gráfico 4).

Gráfico 4. Prevalência de diagnósticos em relação às idades

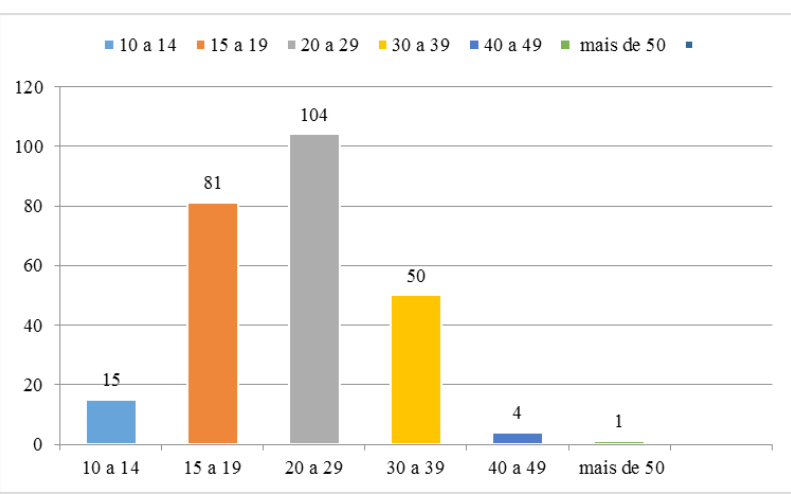

O tipo de exame mais realizado para o diagnóstico da SOP, foi o transvaginal, com 173 mulheres que o realizaram (Gráfico 5).

Gráfico 5. Tipo de exame ultrassonográfico

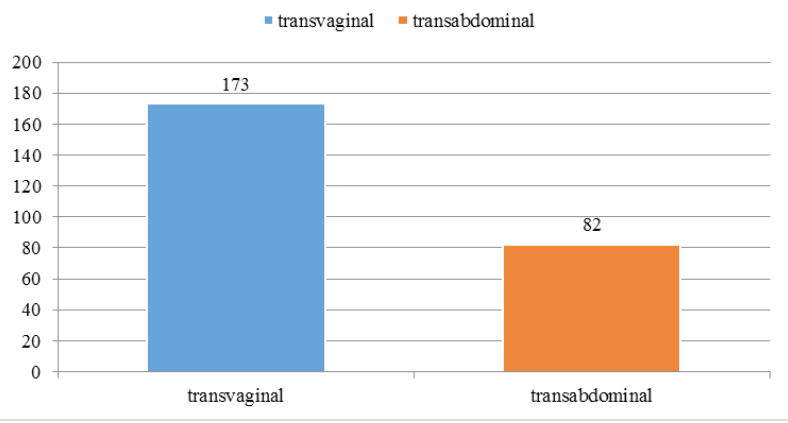

Em relação as formações císticas mais comuns, foi encontrado uma enorme prevalência de casos com ovários apresentando pequenos folículos esparsos, predominando na periferia, esses são cistos que surgiram pelo fato de não ter tido ovulação e o folículo maduro regrediu. E alguns poucos casos, totalizando 26 pacientes, com formação cística diferente da mais comum encontrada. Sendo 15 apresentando formação de cisto lúteo, 7 com a especificação de cistos em forma de "colar de pérolas", 2 com cistos unilaterais e 2 pacientes com múltiplas formações císticas.

$\mathrm{O}$ risco relativo para o volume uterino aumentado na faixa etária de 10 a 19 anos foi 88,65 vezes maior que o seu desenvolvimento normal, nessa mesma faixa etária tanto o volume ovariano direito quanto o esquerdo foram 100 vezes maiores.

Enquanto o risco relativo na faixa etária de 20 a 49 anos em relação ao volume uterino foi de 1,89 vezes maior; o volume ovariano direito foi de 87,97 vezes maior; e o volume ovariano esquerdo foi de 83,54 vezes maior.

\section{DISCUSSÃO}

A síndrome do ovário policístico é caracterizada pelo aparecimento de cistos de origem folicular, que acarretam uma série de sintomas e sinais que atrapalham a vida das portadoras. Na presente pesquisa todas as mulheres estudadas tinham a SOP, e os dados encontrados foram as associações de aumento do volume ovariano, mostraramse mais significativas em mulheres jovens, podendo estar relacionada à resistência periférica a insulina (RI), visto que a SOP é fator de risco para intolerância à glicose. Acreditase que a RI cause a diminuição da concentração da leptina e resistina nos adipócitos das mulheres com SOP, a leptina parece ter efeito direto na esteroidogênese ovariana ${ }^{12}$ e no aumento da secreção hormonal.

É possível afirmar que tal aumento está relacionado com a característica da SOP, pois o grande número de cistos induz uma maior produção hormonal. Sabe-se que as camadas foliculares degeneram-se por ação do infiltrado leucocitário, porém as camadas da teca interna, que são as grandes produtoras hormonais de esteroides e androstenediona, permanecem intactas e continuam a produção normal dos hormônios, desencadeando um episódio de hiperandrogenia, pois vários cistos foliculares atrofiados estão produzindo conjuntamente ${ }^{11}$. Essa hiperprodução hormonal favorece as manifestações clínicas dermatológicas como hirsutismo, acne, alopecia androgenética e acantose nigricante ${ }^{15}$, além de aumentar o risco de desenvolvimento de síndrome metabólica principalmente associada a $\mathrm{RI}^{9}$. Esses sintomas podem se apresentar desencadeando transtornos psicológicos e sociais nas portadoras de $\mathrm{SOP}^{4}$. Em relação aos resultados dos tipos císticos encontrados não fugiram do comum, visto que segundo o estudo experimental de Pedroso et al. ${ }^{12}$, as formações císticas mais encontradas em casos de síndrome do ovário policístico são as de pequenos folículos esparsos.

\section{CONCLUSÃO}

Conclui-se que dentre todas as mulheres avaliadas, de forma geral, as alterações mais comuns estão nas adolescentes, na faixa de 10 a 19 anos, são elas que apresentam os principais sintomas, os aumentos ovarianos e uterinos mais expressivos.

Também foi verificado que a idade mais comum para os diagnósticos feitos no município de Paracatu - MG, foram em mulheres com idades entre 20 a 29 anos, ou seja, uma idade bem tardia para o diagnóstico. Além disso, chamou a atenção a quantidade de casos descobertos, no período de 2009 ao primeiro semestre de 2016, de mulheres portadoras da síndrome, um número consideravelmente grande, e mesmo assim o conhecimento populacional em 
Silva LS, et al. Avaliação da incidência da SOP por clínica e ultrassonografia, em residentes de Paracatu-MG.

relação a SOP e aos seus sintomas mais comuns é pouco.

Portanto, percebeu a necessidade de se divulgar ainda mais a síndrome do ovário policístico para que mais

\section{REFERÊNCIAS}

1. Marcondes JAM, Barcellos CRG, Rocha MP. Dificuldades e armadilhas no diagnóstico da síndrome dos ovários policísticos. Arq Bras de Endocrinol Metab. 2011;55(1):6-15. doi: http://dx.doi.org/10.1590/S0004-27302011000100002.

2. Faria FR, Gonçalves VSS, Faria ER, Gusmão LS, Cecon RS, Franceschini SCC, Priore SE. Síndrome do ovário policístico e fatores relacionados em adolescentes de 15 a 18 anos. Rev Assoc Med Bras. 2013;59(4):341-6. doi: http://dx.doi. org/10.1016/j.ramb.2013.02.003.

3. Sousa RML, Chein MBC, Silva DSM, Dutra MB, Navarro PAAS, Neto JAF, Brito LMO. Perfil metabólico em mulheres de diferentes índices de massa corporal com síndrome dos ovários policísticos. Rev Bras Ginecol Obstet. 2013;35(9):413-20. doi: http://dx.doi.org/10.1590/S010072032013000900006 .

4. Correia D. Análise Computacional de Imagens do Ovário no diagnóstico de síndrome do ovário policístico [dissertação]. Portugal: Faculdade de Engenharia da Universidade do Porto; 2016 [citado 10 out. 2017]. Disponível em: https:// web.fe.up.pt/ tavares/downloads/publications/relatorios/ Diogo_Correia-TP.pdf.

5. Moreira SNT, Sá JCF, Costa EC, Azevedo GD. Qualidade de vida e aspectos psicossociais da síndrome dos ovários policísticos: um estudo quali-quantitativo. Rev Bras Ginecol Obstet. 2013;35(11):503-10. doi: http://dx.doi.org/10.1590/ S0100-72032013001100005.

6. Costa LOBF, Viana AOR, Oliveira M. Prevalência da síndrome metabólica em portadoras da síndrome dos ovários policísticos. Rev Bras Ginecol Obstet. 2007;29(1):10-17. doi: http://dx.doi.org/10.1590/S0100-72032007000100003.

7. Rehme MFB, Pontes AG, Goldberg TBL, Corrente JE, Pontes A. Manifestações clínicas, bioquímicas, ultrassonográficas e metabólicas da síndrome dos ovários policísticos em adolescentes. Rev Bras Ginecol Obstet. 2013;35(6):249-54. doi: http://dx.doi.org/10.1590/S0100-72032013000600003.

8. González F, Kirwan JP, Rote NS, Minium J. Glucose ingestion stimulates atherothrombotic inflammation in polycystic ovary mulheres sejam diagnosticadas precocemente e tenham uma maior qualidade de vida, melhor desenvolvimento psicossocial e melhor convivência com a sociedade.

syndrome. Am J Physiol Endocrinol Metab. 2013;304(4):37583. doi: 10.1152/ajpendo.00491.2012.

9. Motta ELA, Domingues TS, Soares Junior JM. O uso de sensibilizadores de insulina no tratamento de infertilidade em pacientes com síndrome dos ovários policísticos (SOP). Rev Bras Ginecol Obstet. 2012;34(3):99-101. doi: http://dx.doi. org/10.1590/S0100-72032012000300001.

10. Moura HHG, Costa DLM, Bagatin E, Sodré CT, Azulay MM. Síndrome do ovário policístico: abordagem dermatológica. An Bras Dermatol. 2011;86(1):111-9. doi: http://dx.doi. org/10.1590/S0365-05962011000100015.

11. Arie WMY, Fonseca AM, Bagnoli VR, Fassolas G, Baracat EC. Síndrome do ovário policístico e metformina: revisão baseada em evidências. Femina. 2009;37(11):586-602. Disponível em: http://files.bvs.br/upload/S/0100-7254/2009/ v37n11/a002.pdf.

12. Pedroso DCC, Melo AS, Carolo AL, Vieira CS, Silva ACJSR, Reis RM. Frequência e fatores de risco para síndrome metabólica em mulheres adolescentes e adultas com síndrome dos ovários policísticos. Rev Bras Ginecol Obstet. 2012;34(8):357-61. doi: http://dx.doi.org/10.1590/ S0100-72032012000800003.

13. Yarak S, Bagatin E, Hassun KM, Parada MOAB, Talarico Filho S. Hiperandrogenismo e pele: síndrome do ovário policístico e resistência periférica à insulina. An Bras Dermatol. 2005;80(4):395-410. doi: http://dx.doi.org/10.1590/S036505962005000400011 .

14. Mauad Filho F, Mauad FM. Coletânea de tabelas aplicadas em ultrassonografia. Ribeirão Preto: EURP - Escola de Ultrassonografia; s.d [citado 10 out. 2017]. Disponível em: https://www.fatesa.edu.br/media/upload/alunos/coletanea-detabelas-aplicadas-em-ultrassonografia-da-eurp.pdf.

15. Lombardi LA, Simões RS, Maganhin CC, Silva CF, Maciel GAR Baracat EC, Soares Júnior JM. Morfologia das células intersticiais de ovários policísticos de ratas: um estudo experimental. Rev Bras Ginecol Obstet. 2012;34(7):323-8. doi: http://dx.doi.org/10.1590/S0100-72032012000700006. 